Courant Mathematics and

Computing Laboratory

U.S. Department of Energy

\title{
Application of Front Tracking to Two Dimensional Curved Detonation Fronts
}

Bruce Bukiet

Research and Development Report

Supported by the Applied Mathematical Sciences

subprogram of the Office of Energy Research,

U.S. Dept. of Energy under Contract DE-AC02-76ER03077

Mathematics and Computers

June 1985

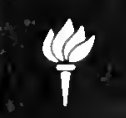

NEW YORK UNIVERSITY 

Courant Mathematics and Computing Laboratory

New York University

Application of Front Tracking to

Two Dimensional Curved Detonation Fronts

Bruce Bukiet

June 1985

Supported by the Applied Mathematical Sciences subprogram of the office of Energy Research, U. S. Department of Energy under Contract No. DE-AC02-76ER0 3077 


\section{DISCLAIMER}

Th1s report was prepared as an account of work sponsored by an agency of the United states Government. Ne1ther the United States Government nor any agency thereof, nor any of their employees, makes any warranty, express or Implied, or assumes any lep,al 11 ab1l1ty or respons 1 b 111 ty for the accuracy, completeness, or usefulness of any Information, apparatus, product, or process disclosed, or represents that 1 ts use would not infrinfe privately owned rights. Reference herein to any speciflc commercial product, process, or service by trade name, trademark, manufacturer, or otherwise, does not necessarliy constitute or $1 \mathrm{mply} 1 \mathrm{ts}$ endorsement, recommendation, or favoring by the United States Government or any apency thereof. The vlews and opinions of authors expressed herein do not necessarliy state or reflect those of the United states Government or any agency thereof.

Printed in U.S.A.

Avatlable from

National Technical Information Service

U.S. Department of Commerce

5285 Port Royal Road

Springfield, VA 22161 


\section{Contents}

Abstract

1. Introduction

2. Elementary Theory

3. Solution of One Dimensional Riemann Problems with Combustion

4. The One Dimensional Computation with

Cylindrical symmetry

5. The Two Dimensional Front Tracking Method 5

6. Results: Strong and CJ Detonations 6

7. Conclusion

8. Acknowledgements

References

Figures 



\title{
Application of Front Tracking to Two Dimensional Curved Detonation Fronts
}

\author{
Bruce Bukiet 12 \\ Courant Institute of Mathematical Sciences \\ New York University \\ New York, N.Y. 10012
}

\begin{abstract}
The method of front tracking is applied to cylindrically symmetric detonations. This two dimensional method is compared to a one dimensional random choice computation in the radial direction which explicitly utilizes the symmetry of the problem. The model of detonations is the Chapman-Jouguet thin flame model. The random choice computation gives a solution of high accuracy of these model equations and is thus taken as exact. We present results demonstrating the high quality of the front tracking solution, even on coarse grids. The solution at the front and in the interior converges linearly to the exact solution. The computation in the interior used a second order method (Lax-Wendroff) and the linear convergence appears to result from the fact that the solution in the interior (i.e. excluding discontinuities) is $C^{1}$ but not $C^{2}$. This is due to "corners" in the solution, located at the edges of a rarefaction wave.
\end{abstract}





\title{
Application of Front Tracking to Two Dimensional Curved Detonation Fronts
}

\author{
Bruce Bukiet 1,2,3 \\ Courant Institute of Mathematical Sciences \\ New York University \\ New York, N.Y. 10012
}

1. Introduction. The application of the method of front tracking to two dimensional fluid flows with curved detonation fronts is examined. We study as test problems two cases of cylindrically symmetric expanding detonation waves, namely a strong detonation and a $\mathrm{CJ}$ detonation. These problems can be solved accurately using a one dimensional random choice computation with operator splitting. We find that the solutions to these problems using front tracking converge linearly to the random choice solutions and give satisfactory answers on moderately coarse grids. We thus conclude that front tracking is a valuable method for computing fluid flows with curved detonation fronts.

2. Elementary Theory. In this section, we describe the elementary theory of one dimensional (planar) Chapman-Jouguet (CJ) detonation waves. The equations of gas dynamics in conservation form are

$$
\boldsymbol{w}_{\mathbf{t}}+\nabla \cdot \mathbf{f}(\mathbf{w})=0
$$

where

$$
w=\left(\begin{array}{c}
\rho \\
m \\
e
\end{array}\right) \text { and } \mathrm{f}(\mathrm{w})=\left(\begin{array}{c}
m \\
\frac{m^{2}}{\rho}+P \\
\frac{m}{\rho}(e+P)
\end{array}\right)
$$

Here $p$ is the density of the gas, $m$ is the momentum density, and $P$ is the pressure. The energy per unit volume, e, may be written as

$$
e=\rho \epsilon+\frac{\rho v^{2}}{2}
$$

1. Supported in part by National Science Foundation Grant Nos. MCS-82-07965 and MCS-83-01255.

2. Supported in part by Army Research Office, Contract No. DAAG-29-84-K0130. 
where $v$ is the velocity and $\epsilon$ is the specific internal energy,

$$
\epsilon=\frac{P}{\rho(\gamma-1)}+q
$$

The gas is polytropic $(\gamma>1)$ and may undergo a chemical reaction in which energy $q$ is released. We assume that the chemical reaction takes place instantaneously and thus that the reaction zone is infinitely thin. Suppose there is a detonation wave connecting constant states labelled by subscripts 0 and 1 . From the conservation of mass, we have

$$
\rho_{1}\left(v_{1}-U\right)=\rho_{0}\left(v_{0}-U\right),
$$

where $U$ is the speed of the detonation wave. From the conservation of momentum, we have

$$
\rho_{1}\left(v_{1}-U\right)^{2}+P_{1}=\rho_{0}\left(v_{0}-U\right)^{2}+P_{0} .
$$

These relations lead to

$$
M^{2}=\frac{P_{1}-P_{0}}{\tau_{0}-\tau_{1}}
$$

where $\tau=\frac{1}{\rho}$ is the specific volume, and $M$ is the the mass flux across the detonation wave. For a right wave, $M_{R}=-\rho_{R}\left(v_{R}-U_{R}\right)$. For a left wave, $M_{L}=\rho_{L}\left(v_{L}-U_{L}\right)$. These equations are the same as those for shock waves.

Equations (2.2) and (2.3) and conservation of energy, expressed by

$$
\left(v_{1}-U\right)\left(e_{1}+P_{1}\right)=\left(v_{0}-U\right)\left(e_{0}+P_{0}\right)
$$

yield

$$
\frac{\gamma_{0} \tau_{0} P_{0}}{\gamma_{0}-1}-\frac{\gamma_{1} \tau_{2} P_{1}}{\gamma_{1}-1}-\left(q_{1}-q_{0}\right)=\frac{\left(P_{0}-P_{1}\right)\left(\tau_{0}+\tau_{1}\right)}{2}
$$

called the Hugoniot relation.

Suppose the gas in state 0 is unburned and that the gas in state 1 is burned. The Hugoniot curve describes in the $(\tau, P)$-plane the states to which the gas in state 0 may be connected by a single combustion wave (See Fig. [1]). The portion of the curve bordering 3. Supported in part by U.S. Dept. of Energy, Contract No. DE-AC02-76ER03077. 
the shaded region corresponds to non-physical solutions, where $M^{2}<0$. The portion of the curve to the right of the shaded region corresponds to deflagrations. The portion to the left corresponds to detonations.

A line drawn from $\left(\tau_{0}, P_{0}\right)$ that is tangent to the detonation portion of the Hugoniot curve divides it into two parts. The upper part corresponds to strong detonations and the lower part to weak detonations. The point of tangency is called the Chapman-Jouguet (CJ) point. At a CJ detonation, the detonation wave moves at sound speed with respect to the gas behind it $[4, p .212]$. Thus,

$$
\rho_{1}^{2} c_{1}^{2}=\rho_{1}^{2}\left(v_{1}-U\right)^{2}=M^{2}=\frac{P_{1}-P_{0}}{T_{0}-T_{1}}
$$

behind a CJ detonation, where $c^{2}=\frac{\gamma P}{\rho}$.

Combining (2.5) and (2.6), we get, after some algebra,

$$
\begin{aligned}
P_{C J}^{2}+ & \left(\frac{2\left(q_{1}-q_{0}\right)\left(\gamma_{1}-1\right)}{\tau_{0}}-\frac{2\left(\gamma_{1}-1\right) P_{0}}{\gamma_{0}-1}\right) P_{C J} \\
& -\frac{2\left(\gamma_{1}-1\right)\left(q_{1}-q_{0}\right) P_{0}}{\left(\gamma_{1}+1\right) \tau_{0}}+\frac{\left(\gamma_{1}-1\right)\left(\gamma_{0}+1\right)}{\left(\gamma_{1}+1\right)\left(\gamma_{0}-1\right)}=0
\end{aligned}
$$

which is solved by the quadratic formula. Thus, the CJ state is completely determined by the state ahead of the detonation. The larger of the two solutions of equation (2.7) is the CJ detonation pressure. The smaller va!ue corresponds to a $\mathrm{CJ}$ deflagration. We get the values of $\rho_{C J}$ and $\tau_{C J}$ from equation (2.6). Also, from (2.6), $M=\rho_{C J} c_{C J}=\gamma_{C J} P_{C J} \rho_{C J}$. We use equations (2.2) and the mass flux equations to get $v_{C J}$ and $U_{C J}$.

3. Solution of One Dimensional Rlemann Problems with Combustion. Consider equation (2.1) with the initial data

$$
\begin{array}{ll}
S_{L}=\left(\rho_{L}, u_{L}, P_{L}\right) & \text { for } x<0 \\
S_{R}=\left(\rho_{R}, u_{R}, P_{R}\right) & \text { for } x>0 .
\end{array}
$$

The solution at a later time will consist, in general, of a right wave, a left wave and a contact. We allow the right (or left) wave to be a shock, rarefaction, strong detonation, CJ detonation or $\mathrm{CJ}$ detonation followed by a rarefaction. Across the contact, the pressure and 
velocity are continuous, though the density may jump. In order to include at least some aspects of quenching in the model, we introduce a critical temperature $\left(T_{c}\right)$ and allow combustion to occur only if the midstate temperature is above $T_{c}$.

We write the equations for the states to which we may connect the left and right states. Since the left and right states connect to states with the same velocity and pressure, the equations are expressed as velocity as a function of pressure. Then the Riemann problem is solved by finding the intersection of these curves. To find the equations for shocks or detonations, eliminate $\rho_{1}$ and $U$ from eqs. (2.2) (2.3) and (2.4), letting $q_{1}=q_{0}$ for shocks. The equations for rarefactions are found using the Riemann invariants

$$
\begin{array}{ll}
\frac{v_{L}}{2}+\frac{c_{L}}{\gamma_{L}-1}=\frac{v_{*}}{2}+\frac{c_{*}}{\gamma_{*}-1} & \text { for a left rarefaction } \\
\frac{v_{R}}{2}-\frac{c_{R}}{\gamma_{R}-1}=\frac{v_{*}}{2}-\frac{c_{*}}{\gamma_{*}-1} & \text { for a right rarefaction }
\end{array}
$$

as well as the isentropic law

$$
\begin{aligned}
& P_{L} \rho_{L}^{-\gamma_{L}}=P_{*} \rho_{*}^{-\gamma_{*}} \quad \text { on the left } \\
& P_{R} \rho_{R}^{-\gamma_{R}}=P_{*} \rho_{*}^{-\gamma_{*}} \quad \text { on the right, }
\end{aligned}
$$

where * denotes the state on the inside of the wave. For a CJ detonation followed by a rarefaction, we find the $\mathrm{CJ}$ state and use the equations for rarefactions, substituting $C J$ for the subscript $L$ or $R$.

We use a Newton method iteration scheme [1] to find the intersection point and thus solve the Riemann problem. The algorithm initially finds a solution with only hydrodynamic waves. If the hydrodynamic midstate temperature $(T=P / P)$ is above some prescribed critical temperature, i.e. the temperature at which the unburned gas burns, and the gas is unburned, then the hydiodynamic solution is replaced by a solution utilizing combustion waves. The random choice method [5] has been applied to reactive gas dynamics in one dimension [3],[6] using the above solution of this Riemann problem. 
4. The One Dimensional Coinputation with Cylindrical Symmetry. With cylindrical symmetry, the equations of gas cynamies (2.1) become

$$
\mathbf{w}_{\mathrm{t}}+\mathbf{f}(\mathbf{w})_{\mathrm{s}}=-\mathbf{S}
$$

where

$$
S=\left(\begin{array}{c}
\frac{m}{r} \\
\frac{m^{2}}{\rho r} \\
\frac{m}{\rho r}(e+P)
\end{array}\right)
$$

and $\mathrm{r}$ is the radius.

Sod [7] developed a method for solving this system using the random choice method and operator splitting. At the start of a time step, we solve the homogeneous system

$$
\mathbf{w}_{t}+\mathbf{f}(\mathbf{w})_{r}=0
$$

by the random choice method. Next, we use the solution of eq. (4.1) as initial data for the system of ordinary differenial equations

$$
\mathbf{w}_{\mathrm{t}}=-\mathbf{S}
$$

For $p \neq 0$, this can be simplified to

$$
\left(\begin{array}{l}
\rho \\
v \\
\rho
\end{array}\right)_{t}=-\left(\begin{array}{c}
\frac{\rho v}{r} \\
0 \\
\frac{\gamma P v}{r}
\end{array}\right),
$$

which is solved analytically. We use a planar CJ thin flame detonation as described in $\$ 3$, in the cylindrical solution. This approximation is exact in the limit of large radius.

5. The T ғo Dlmenslonal Front Tracking Method. We present a brief summary of front tracking [2] which models two dimensional fluid flow. A front consists of a system of curves. These curves, which are usually associated with physical waves of discontinuity, evolve with the solution. 
The portion of the computational region away from the front is called the interior. In the interior, we solve an initial/boundary-value problem, where the curves play the role of boundaries. In our computations, an operator split Lax-Wendroff method is used to solve eq. (2.1) in the interior.

The propagation of the front requires both the movement and updating of the states on the front. Eq. (2.1) is written in terms of its normal and tangential derivatives and operator splitting is employed. First, a non-local Riemann problem for the detonation theory of $\$ 3$ is solved in the normal direction, propagating the discontinuity. Then, the solution of the normal propagation is taken as initial data for the tangential propagation. A one dimensional Lax-Wendroff scheme is used for the tangential equations.

6. Results: Strong and CJ Detonations. In this section, we present the results of computations for expanding circular (cylindrically symmetric) and elliptical strong detonations. The circular geometry was chosen for our test problem because it is essentially a one dimensional problem and the solutions by front tracking can therefore be compared to solutions by the random choice method. The first sequence of runs models a strong detonation. The second sequence shows the transition from strong detonation to CJ detonation. The two dimensional computations were done on the following grids: 5 by 5,10 by 10,20 by 20,40 by 40 and 80 by 80 . In these computations, we tracked the contact as well as the detonation. The one dimensional computations used 1500 points in the radial direction, and are taken as giving the exact solution, within the modelling assumptions of the planar strong and CJ detonation theory.

In Figs. 2a-f we present the results of computations in which the pressure inside the initial circle is 100 times the pressure outside. The density is uniform and the gas releases 92.65\% of its internal energy upon combustion. The pressure and heat release were chosen to yield a strong detonation from the start of the computation through the time step analyzed. Figs. 2 a-e refer to a front tracking computation on a 40 by 40 grid. Fig. 2 a shows the positions of the contact (inner quarter circle) and the detonation (outer quarter circle) at the start of the run. The initial pressure profile is shown in Fig. 2b. Fig. 2c shows the positions of the contact and the detonation at the time step analyzed. The corresponding pressure profile is shown in Fig. 2d. In this profile, the solid curve is the result of the one dimensional 
calculation, while the vertical error bars show the range of pressure values in the front tracking solution at fixed radii, indicating angular dependence. In Fig. $2 \mathrm{e}$, we present the detona. tion wave speed plotted against time for the one dimensional run (solid curve) along with the range of speeds of the detonation in the two dimensional computation (vertical bars). We note that the front tracking detonation speed error is less than $\% \%$. Fig. $2 \mathrm{f}$ shows the convergence of the front tracking solution to the random choice solution. Since the solution is not smooth, the Lax-Wendroff method used in the interior is only linearly convergent. For each grid, we compute the pressure error, in percent, at the front and in the interior. These errors are plotted against the reciprocal of the number of grid points in the $\mathbf{x}$ and $\mathbf{y}$ directions. Since we use the same number of grid points $(N)$ in each direction and the computational region is .5 by .5 , the abscissa is $\frac{1}{N}=2 \Delta x=2 \Delta y$. The percent error in the integral of pressure (\#'s) over the computational region (interior error), the range of errors of pressure, in percent, (error bars) behind the front and the percent error of the average value of pressure behind the front (asterisks) are shown in Fig. 2f. The lines show the errors going linearly to 0 as $\Delta x=\Delta y \rightarrow 0$.

Similarly, in Figs. 3a-d, we show results for an initial pressure ratio inside to outside of 68. In these runs, the parameters were chosen to yield a $\mathrm{CJ}$ detonation for approximately three-fourths of the elapsed time at the time analyzed in these figures. The tenfold smaller errors in detonation speed in Fig. $3 b$ result from the smaller variations in the speeds in this 40 by 40 run. In Figs. 3c and 3d, we present separately the interior error and front error for pressure.

Finally, we present an example for which a one dimensional method cannot be used. In this example, we find hot spots behind the front in regions of small curvature and cold spots in corresponding regions of large curvature. We consider an elliptical expanding detonation with the same initial states as those in the strong detonation runs above. The initial lengths of the major and minor axes are .3 and .15 for the detonation wave and .29 and .145 for the contact. Fig. 4 shows pressure contours and the waves just before the detonation wave breaks through the boundary on a 30 by 30 grid. We see that the pressure is higher behind the flatter portion of the detonation wave than behind the rounder portion of the 
wave.

7. Conclusion. We have shown that for modelling fluid flows with cylindrically symmetric detonation waves, the solution by the method of front tracking converges linearly to the exact (one dimensional random choice) solution. We believe these results validate front tracking for the computation of two dimensional fluid flows with curved detonation fronts.

8. Acknowledgements. The author thanks J. Jones, D. Marchesin and B. Plohr for their assistance on the work that led to this paper. He thanks O. McBryan for providing the computer graphics routines. He also thanks J. Glimm for his inspiration and guidance in this project. 


\section{REFERENCES}

[1] B. BLKIET, "Ph.D. Thesis," In preparation, 1985.

[2] I.L. Cherv, J. Glad, O. MCBRyan,B. Plohr, "Front Tracking for Gas Dynamics," DOE Research and Development Report DOE/ER/03077-223, May, 1984, To appear, J. Comp. Phys.

[3] A. J. CHORIN, "Random Choice Methods with Applications to Reacting Gas Flows," J. Comp. Phys., vol. 25, pp.253-272, 1977.

[4] R. COURANT AND K. O. FRIEDRICHS, "Supersonic Flow and Shock Waves," SpringerVerlag, New York, 1976.

[5] J. GLIMM, "Solutions in the Large for Nonlinear Hyperbolic Systems of Equations," Comm. Pure Appl. Math. vol. 18, pp.695-715, 1965.

[6] T. SAITO AND I. I. GLass, "Application of Random-Choice Method To Problems in Gasdynamics," Prog. Aerospace Sci., vol. 21, pp.201-247, 1977.

[7] G. A SOD, "A Numerical Study of a Converging Cylindrical Shack," J. Fluid Mech., vol. 83, pp.785.794, 1977. 


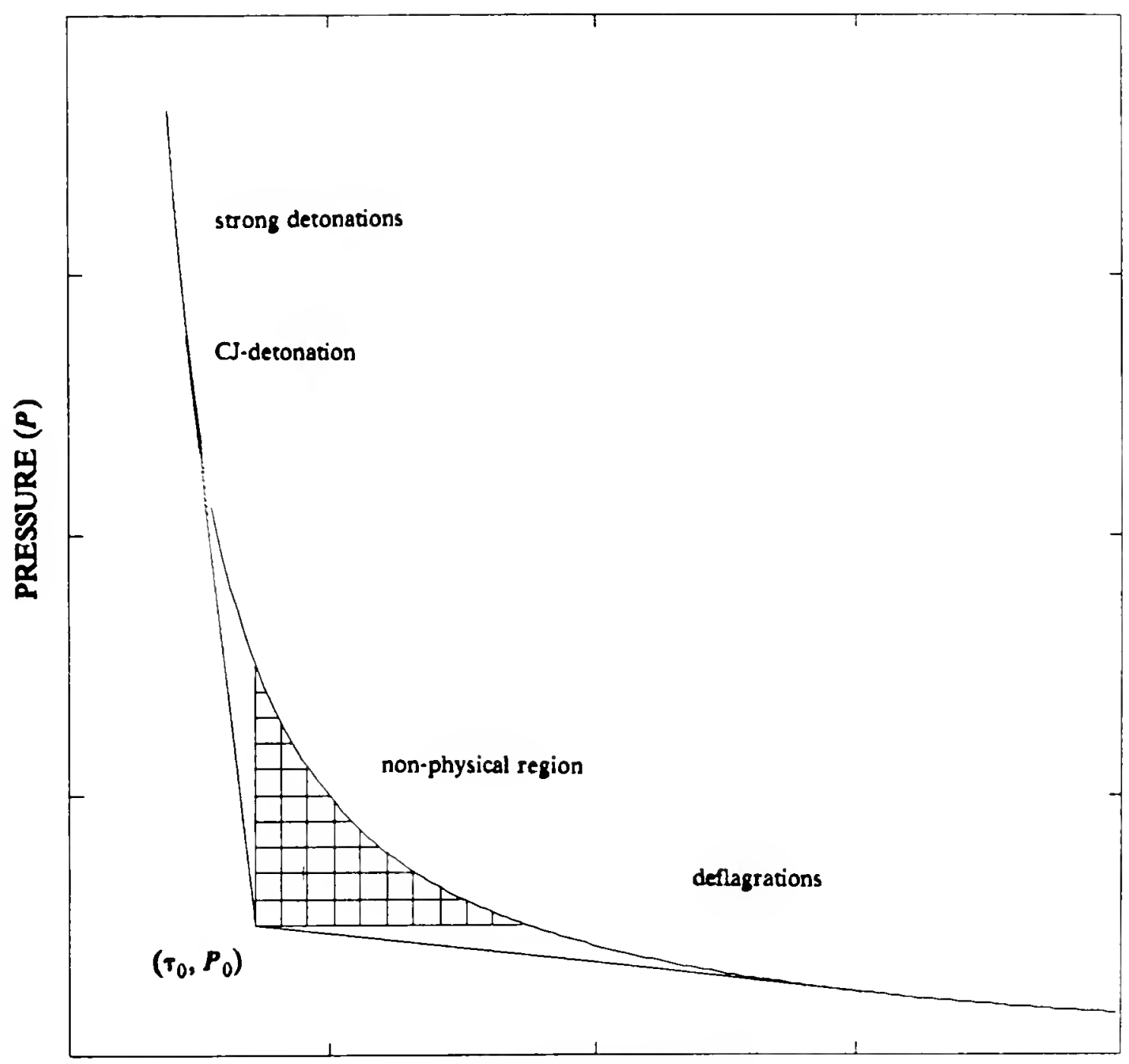

SPECIFIC VOLUME $(\tau)$

Fig. 1. A Hugoniot curve showing the states to which the state $\left(\tau_{0}, P_{0}\right)$ may be connected by combustion waves. The shaded region corresponds to non-physical solutions. The lines connecting $\left(\tau_{0}, P_{0}\right)$ to the $C J$ points, and thus cangent to the Hugoniot curve, are drawn. 


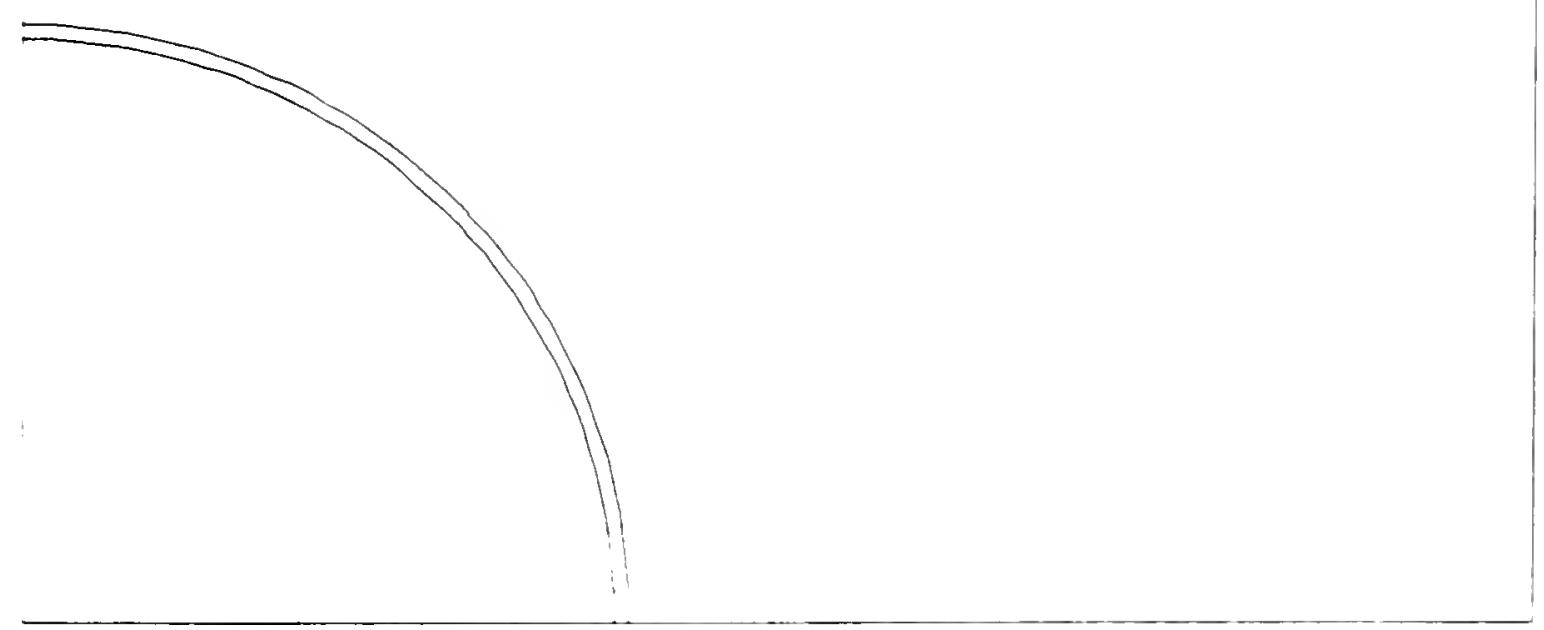

Fig. 2. The strong detonation. 8. The initial strong detonation wave (ouser circle) and the contact discontinulty for a cylindrically symmetric computation. The initial conditions are uniform density, zero velocity, and a circular pressure discontinuity at radius .2, with ratio inside to outside of 100 . The heat released upon combustion is $92.65 \%$ of the internal energy of the unburned gas. The initial pasition of the contact is radius 195. The initial state between the waves is that behind a planar detonation wave with the above initial data. 


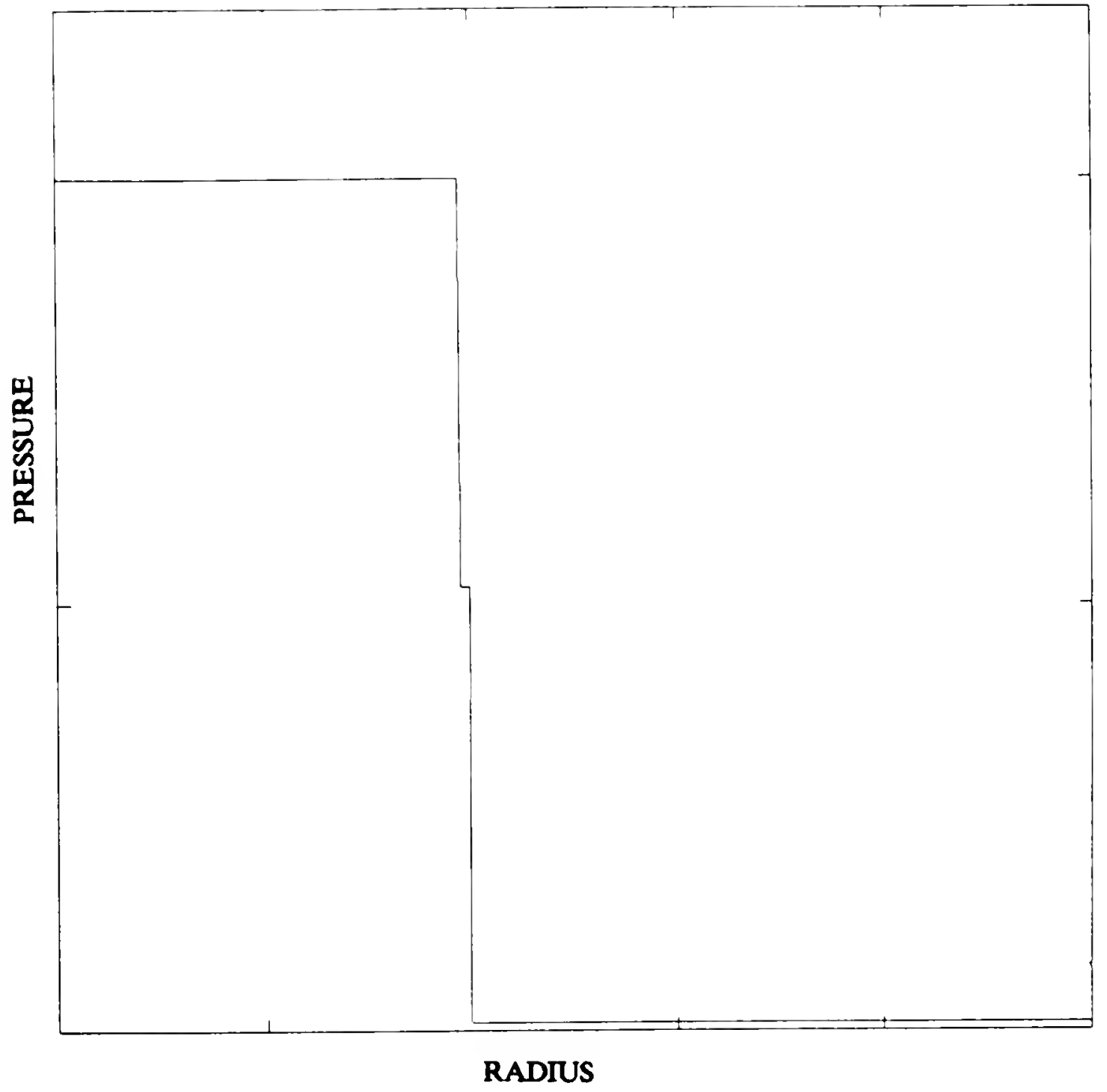

Fig. 2b. A plot of pressure vs. radius at time $=0$. 


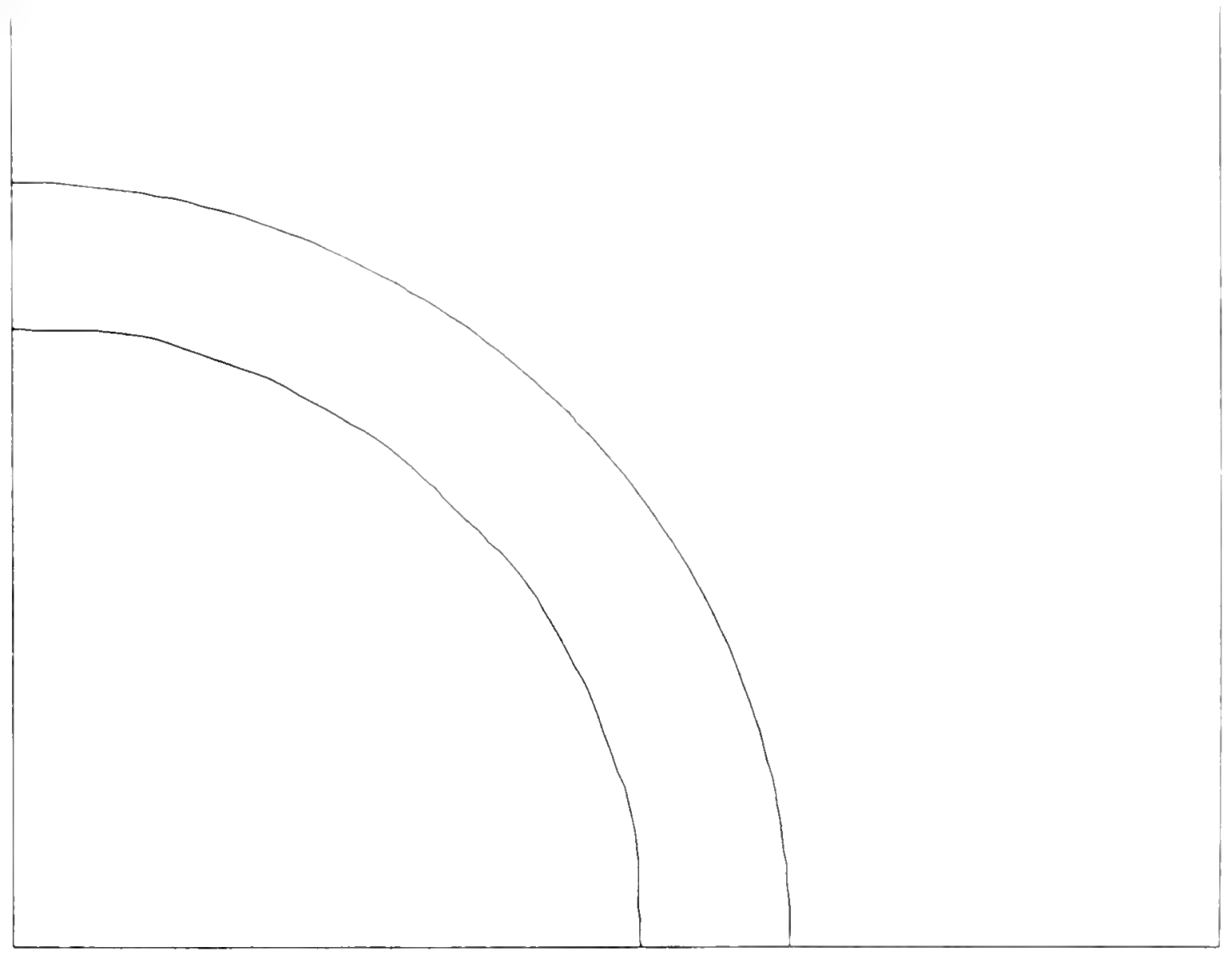

Fig. 2c. The detonation wave and the contact at the time step analyzed in Figs. 2d and $2 \mathrm{f}$. The detonation wave now has radius appraximately 36 . 


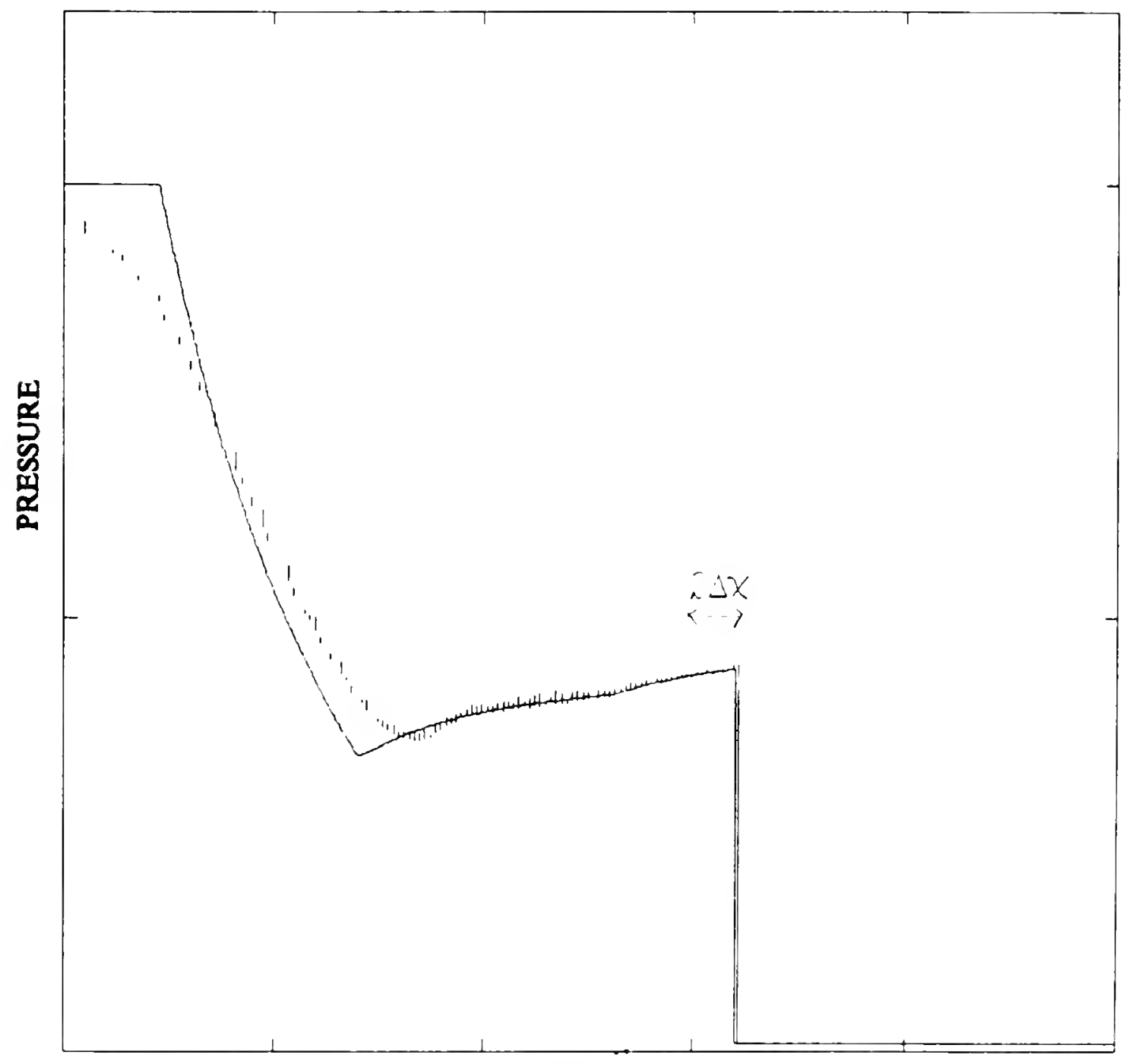

\section{RADIUS}

Fig. 2d. A plot of pressure vs. radius corresponding to Fig. $2 \mathrm{c}$ is shown. The solid curve shows the results obtained by the one dimensional random choice computation. The vertical lines represent the range of pressure values in the two dimensional front racking solution at a fixed radius as the angle varies on $a 10$ by 10 grid. Thus, the vertical lines show the angular dependence in the solution. 


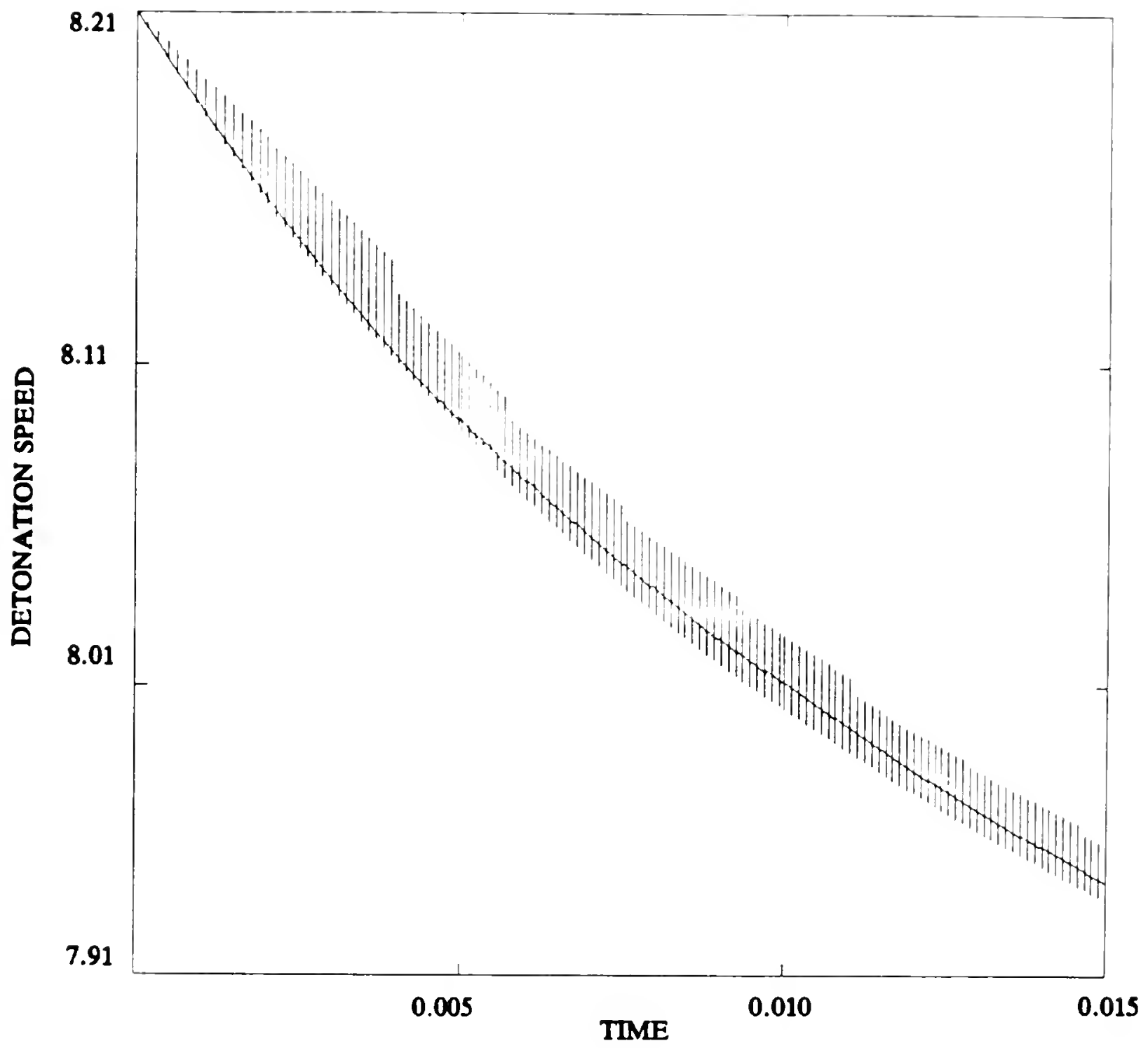

Fig. 2e. A plot of detonation speed vs. time for the computation on a 40 by 40 grid. The solid curve shows the speed of the detonation wave in the one dimensional calculation. The vertical lines represent the range of values of the speed of the detonation in ine two dimensional calculation. The maximum error ( $\max \frac{\left|U_{2 d}-U_{I d}\right|}{U_{I d}}$ ) is less than $4 \%$. 
$30 \%$

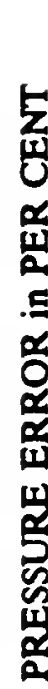

$20 \%$

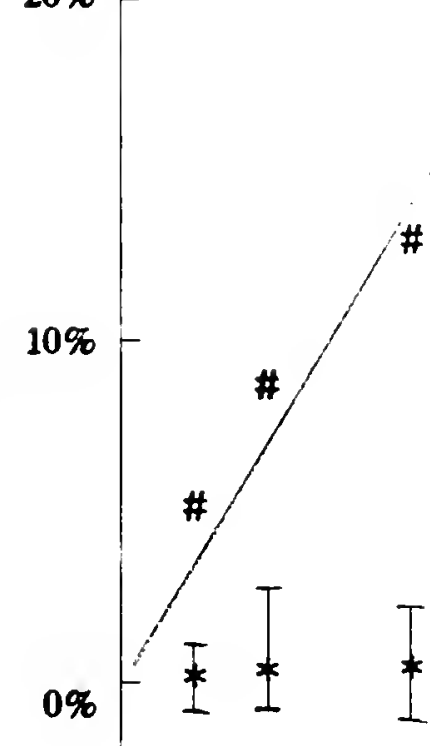

* = interior error

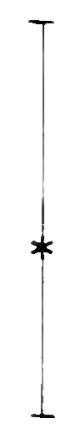

1 = front error

* = average front error

$-10 \% \quad N=80 N=40 \quad N=20$

$\mathbf{N}=\mathbf{1 0}$

$\mathbf{N}=\mathbf{S}$

$$
\frac{1}{N}=2 \Delta x=2 \Delta y
$$

Fig. 2f. Convergence of the front and interior schemes. The pressure errors in the interior and at the front are shown for $N \times N$ grids at the time indicated by Fig. 2c. The \# signs represent the interior error, where

$$
\text { Interior Error }=100 \% \times \frac{\int_{0}^{1} \int_{0}^{\infty}\left|P_{2 d}-P_{I d}\right| d x d y}{\int_{0}^{1} \int_{0}^{1} P_{\text {lnttal dara }} d x d y}
$$

The front error (error bars) gives the range of the errors at the front, defined as

$$
\text { Front Error }=100 \% \times \frac{P_{2 d}-P_{1 d}}{[P]} \text {, }
$$

where $[P]$ is the pressure jump at the front in the one dimensional computation at the same time. The asterisks represent the error of the average pressure behind the front, namely

Front Error (average pressure) $=100 \% \times \frac{P_{2 d \text { coverage }}-P_{1 d}}{[P]}$ 


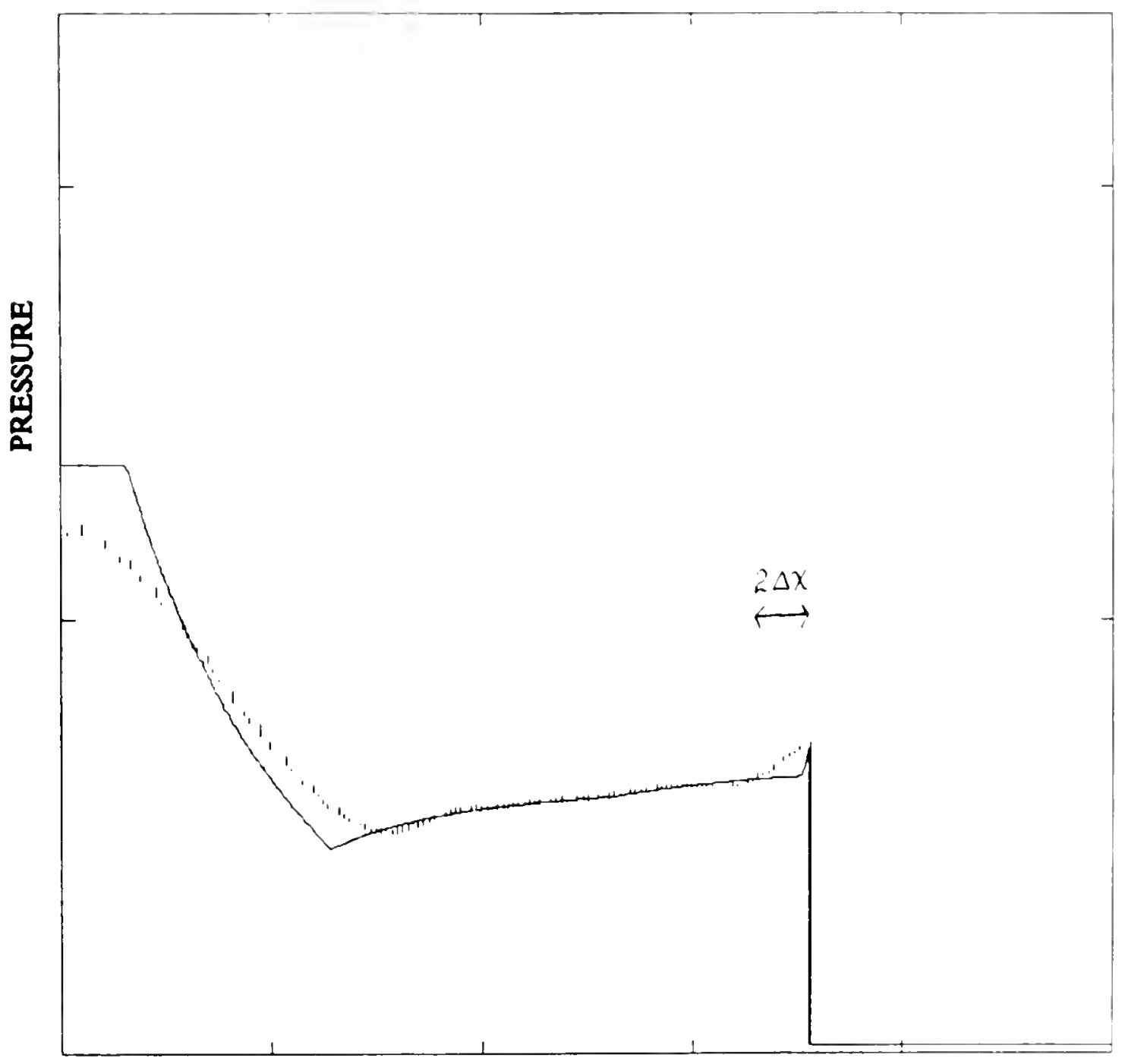

\section{RADIUS}

Fig. 3. The CJ detonation. a. A plot of pressure vs, radius for a run similar to that described in Figs. 2a-e but for a pressure ratio of 68 is shown. The solid curve and the vertical lines represent the one and two dimensional results, as explained in the caption to Fig. 2d. The initial conditions are uniform density, zero velocity, and a circular pressure discontinuity at radius .2. The heat released upon combustion is $92.65 \%$ of the internal energy of the unburned gas. The initial position of the contact is radius.195. The initial state between the waves is that behind a planar detonation wave with the above initial data. 


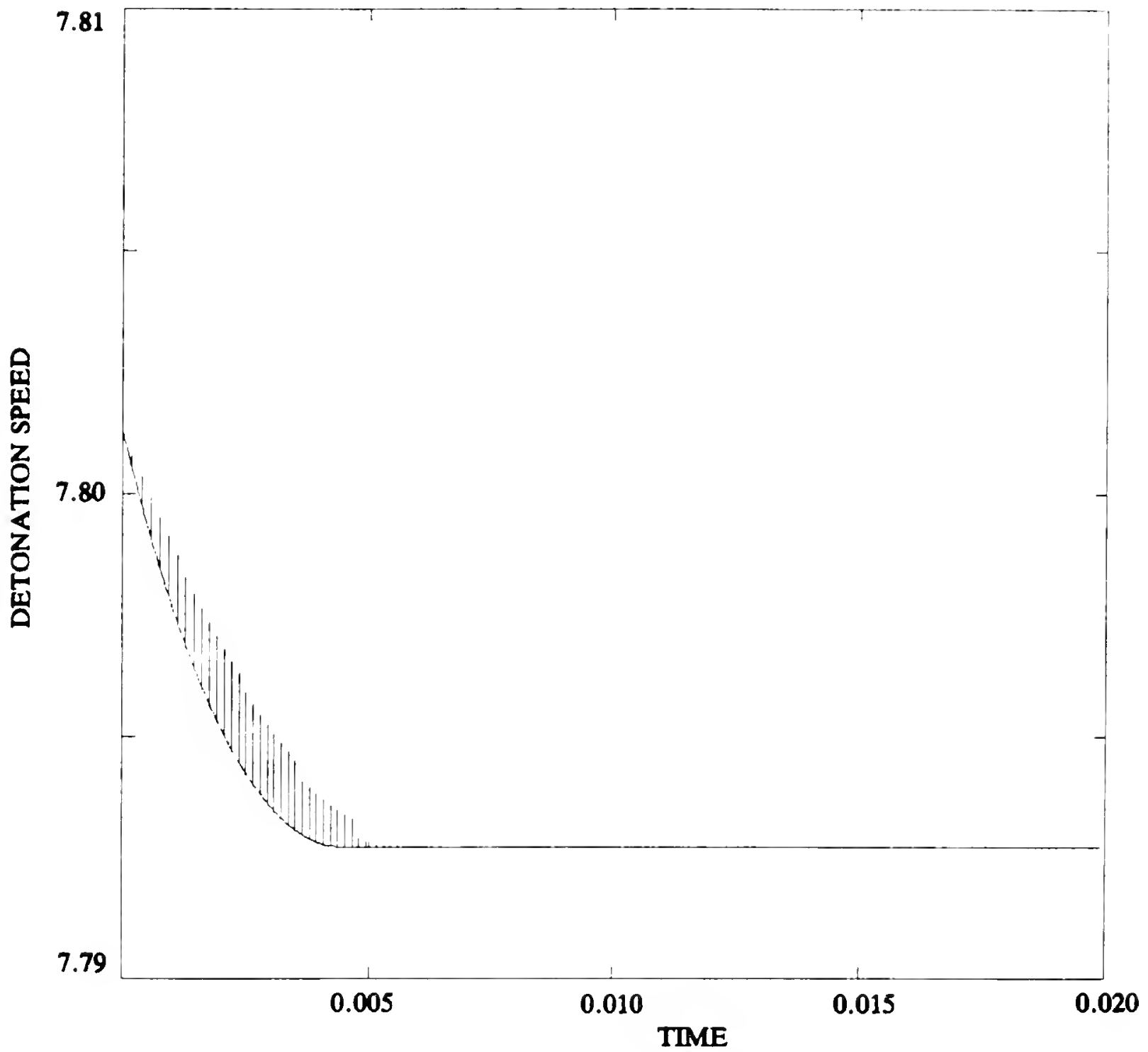

Fig. 3b. A plot of detonation speed vs. time for the computation on a 40 by 40 grid. The solid curve shows the speed of the detonation wave in the one dimensional calcula. tion. The vertical lines represent the range of values of the speed of the detonation in the two dimensional calculation. The maximum error $\left(\max \frac{\left|U_{2 d}-U_{1 d}\right|}{U_{1 d}}\right)$ is less than $.05 \%$. 


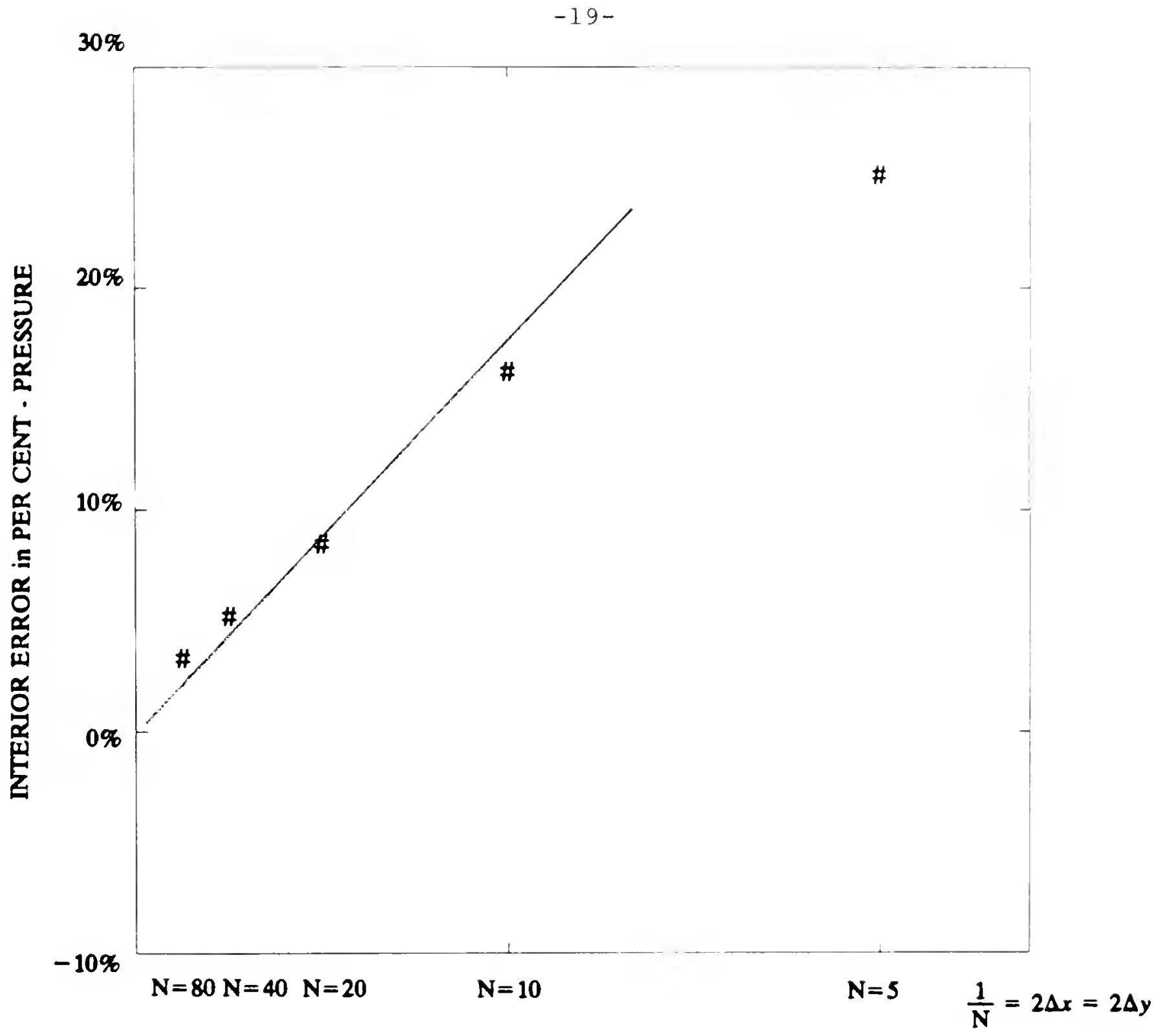

Fig. 3c. Convergence of the interior scheme. The pressure errors in the interior are shown for NxN grids for the time represented by Fig. 3a. The error is defined as in Fig. $2 \mathrm{f}$. 


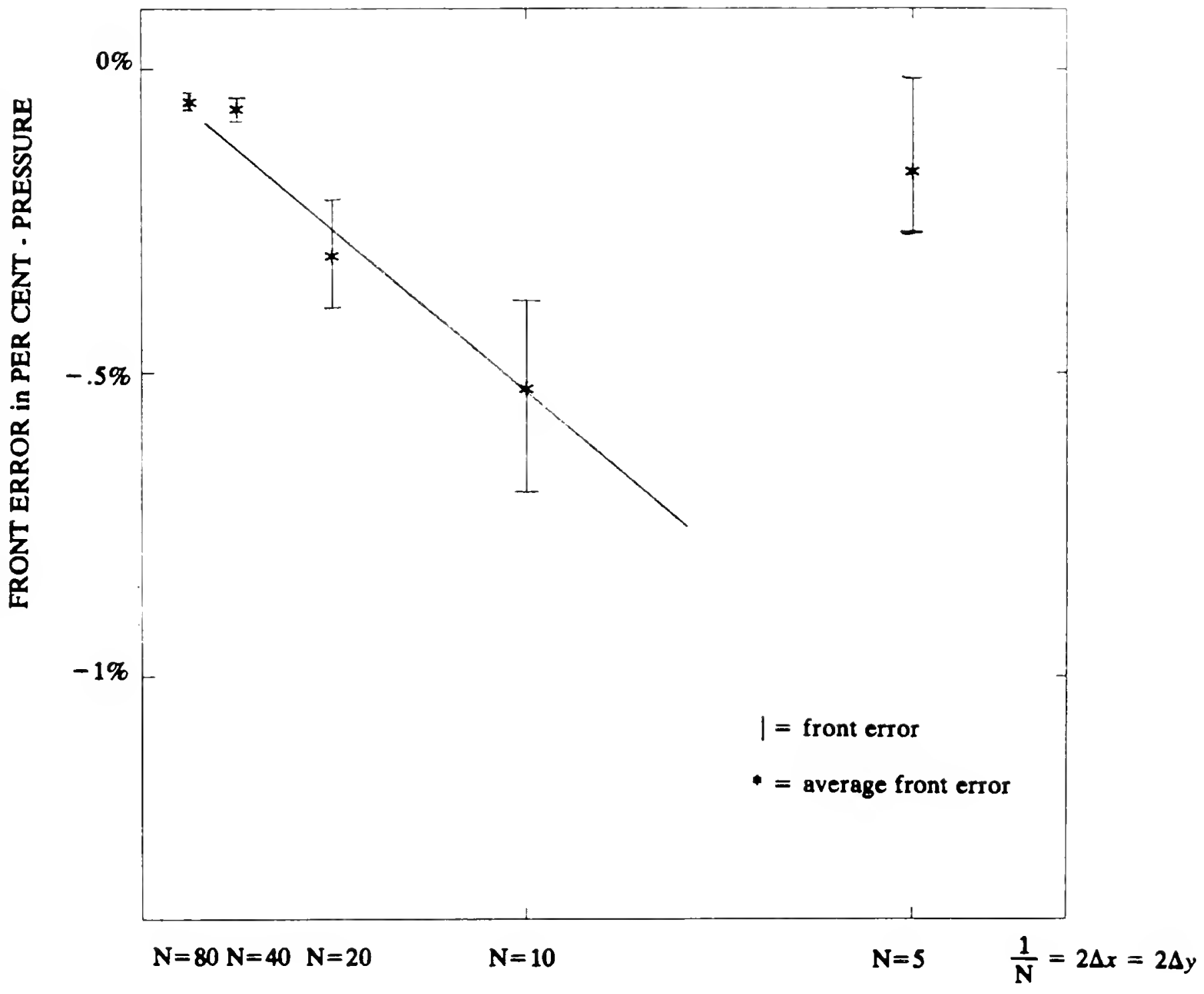

Fig. 3d. Convergence of the front scheme. The front error is shown for $N x N$ grids for the time represented by Fig. 3a. The front error (error bars) gives the range of the errors at the front. The asterisks represent the error of the average pressure behind the front. These errors are as defined in the caption to Fig. 2f. 


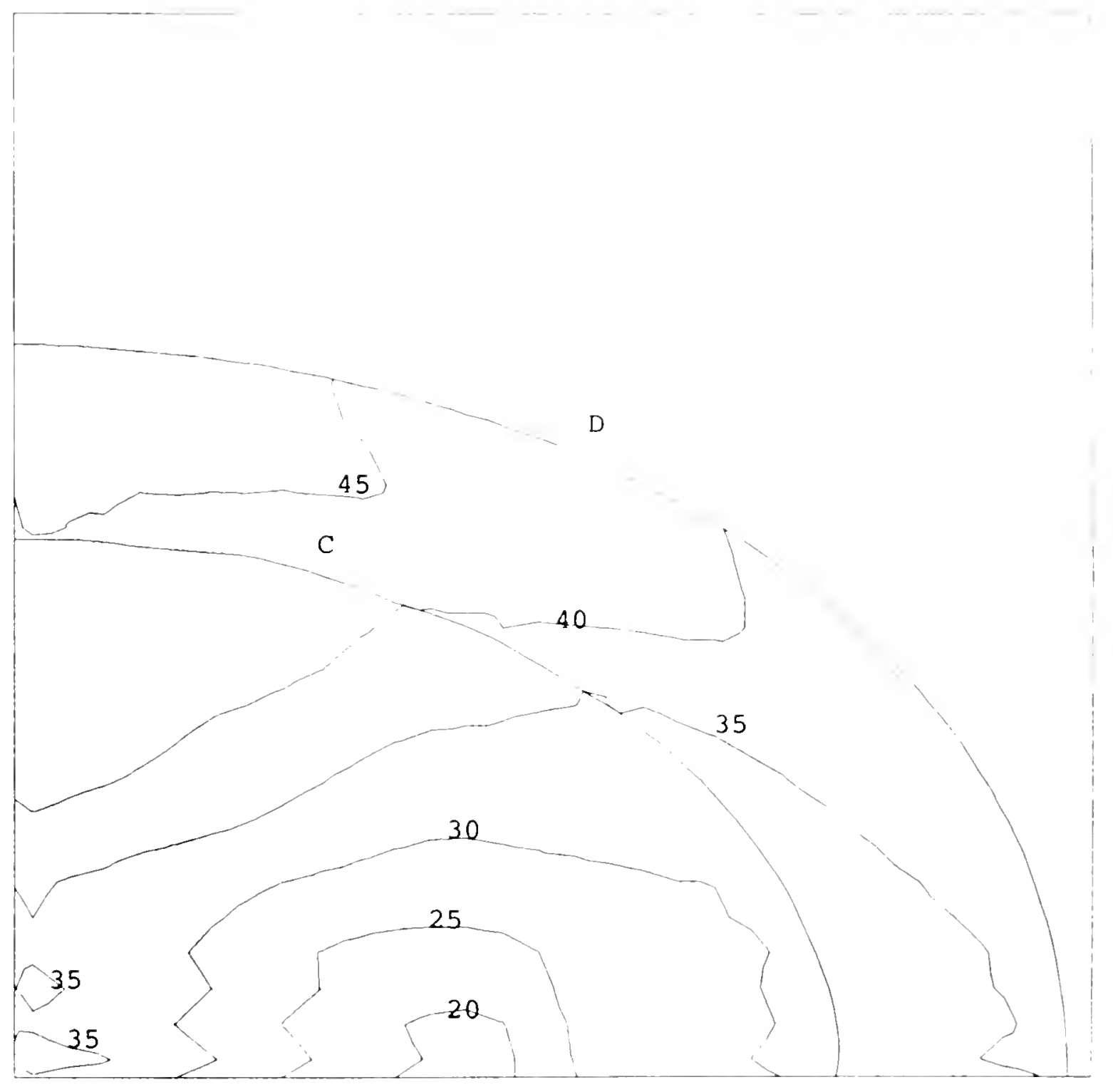

Fig. 4. Pressure contours are shown for a computation of an elliptical expanding detonation on $a 30$ by 30 grid. Also shown are the detonation wave $(D)$ and the contact $(C)$. 


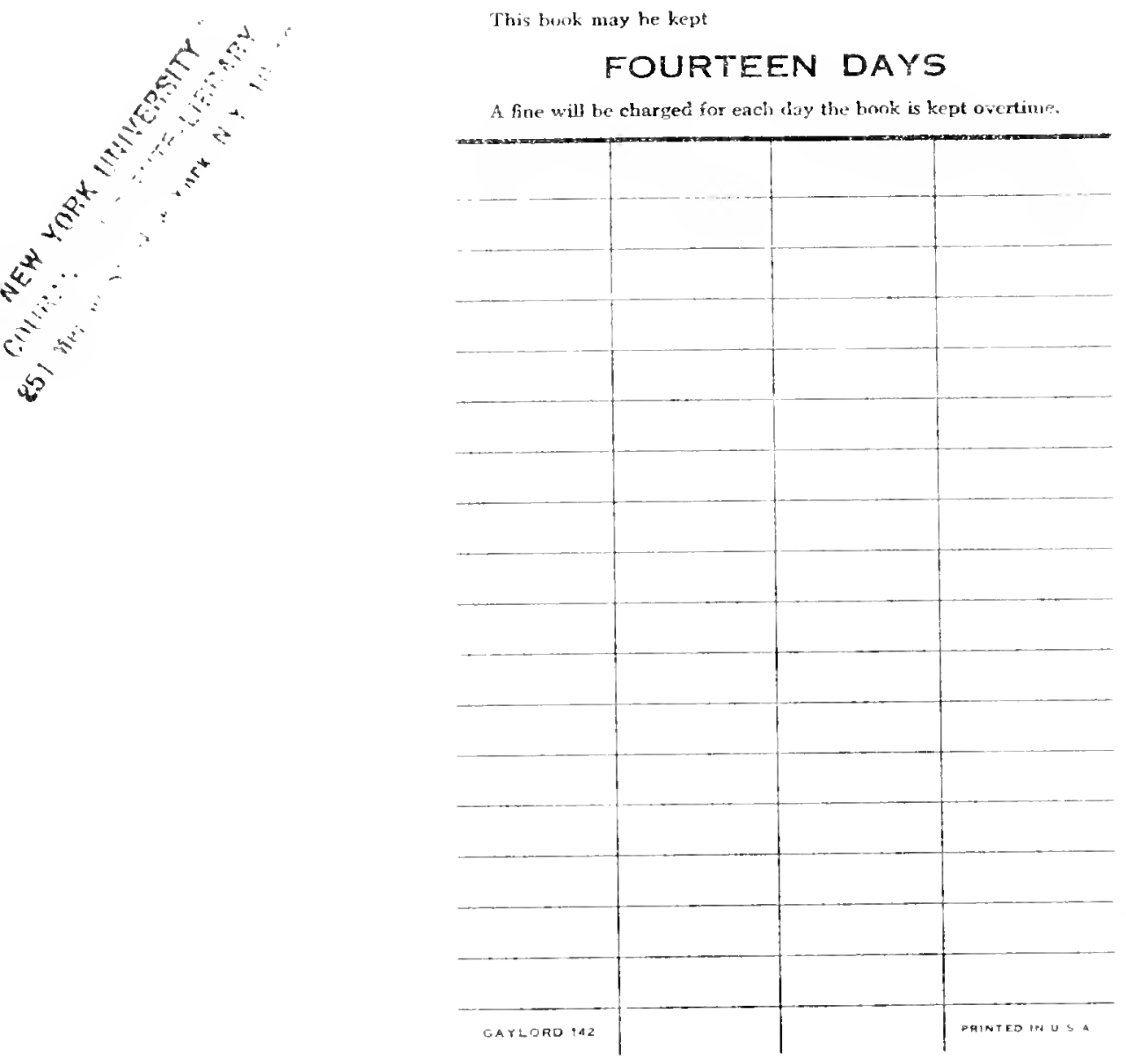


NYU DOE/ER/03077-26I c。I

Bukiet

Application of front...

LIBRARY

N.Y.U. Courant Institute of Mathematical Sciences

251 Mercer St.

New York, N. Y. 10012 
